Bull. Austral. Math. Soc.

$47 \mathrm{~A} 15,06 \mathrm{~B} 15,47 \mathrm{C} 05,06 \mathrm{D} 10$

VoL. 58 (1998) [245-260]

\title{
ABSTRACT REFLEXIVE SUBLATTICES AND COMPLETELY DISTRIBUTIVE COLLAPSIBILITY
}

\author{
W.E. Longstaff, J.B. Nation and Oreste Panaia
}

\begin{abstract}
There is a natural Galois connection between subspace lattices and operator algebras on a Banach space which arises from the notion of invariance. If a subspace lattice $\mathcal{L}$ is completely distributive, then $\mathcal{L}$ is reflexive. In this paper we study the more general situation of complete lattices for which the least complete congruence $\Delta$ on $\mathcal{L}$ such that $\mathcal{L} / \Delta$ is completely distributive is well-behaved. Our results are purely lattice theoretic, but the motivation comes from operator theory.
\end{abstract}

A subspace lattice $\mathcal{L}$ is reflexive if it is the set of invariant subspaces of some collection of operators, which is equivalent to $\mathcal{L}=\operatorname{Lat} A \lg \mathcal{L}$ in the notation given below. In [7], the first author proved that any completely distributive subspace lattice is reflexive (generalising Halmos [4]). The crucial lemma in this proof describes, for a given subspace lattice $\mathcal{L}$, the lattice $\widehat{\mathcal{L}}$ of subspaces invariant under the rank-1 operators leaving every member of $\mathcal{L}$ invariant. The map $\mathcal{L} \mapsto \widehat{\mathcal{L}}$ is a closure operator on subspace lattices, and can be described in purely lattice theoretic terms. As $\widehat{\mathcal{L}}$ is a reflexive lattice, we can think of this as an abstract way of constructing reflexive lattices. (Though not every reflexive subspace lattice is $\widehat{\mathcal{L}}$, for some subspace lattice $\mathcal{L}$. Since reflexivity is not a lattice-theoretic invariant [10], it can have no lattice-theoretic characterisation.)

It is interesting then to look for classes of lattices where the map $\mathcal{L} \mapsto \widehat{\mathcal{L}}$ is wellbehaved, for this will allow us to construct many examples of reflexive subspace lattices. This in turn leads us to consider the least complete congruence $\Delta$ on $\mathcal{L}$ such that $\mathcal{L} / \Delta$ is completely distributive. The general description of $\Delta$ is not very enlightening, but under certain circumstances it simplifies considerably. With these same conditions, the map $\mathcal{L} \mapsto \widehat{\mathcal{L}}$ becomes quite tractable. The corresponding class of lattices, called $\mathcal{G}$-lattices, and its subclass of $\partial$-lattices, will be studied in the latter part of the paper.

\section{BACKGROUND}

In this section we shall assemble the relevant background and terminology for both lattice theory and operator theory. Throughout, we shall be working with complete Received 11th February, 1998

Copyright Clearance Centre, Inc. Serial-fee code: 0004-9729/98 \$A2.00+0.00. 
lattices, whose operations are denoted by $\Lambda$ and $\vee$, with a least element 0 and greatest element 1 . We adopt the usual conventions that $\bigvee \emptyset=0$ and $\wedge \emptyset=1$.

If $\mathcal{L}$ and $\mathcal{K}$ are complete lattices, a map $h: \mathcal{L} \rightarrow \mathcal{K}$ is a complete homomorphism if $h\left(\bigvee_{i \in I} x_{i}\right)=\bigvee_{i \in I} h\left(x_{i}\right)$ and $h\left(\bigwedge_{i \in I} x_{i}\right)=\bigwedge_{i \in I} h\left(x_{i}\right)$, for any index set $I$. The kernel of a complete homomorphism is called a complete congruence. Thus every complete congruence is an equivalence relation $\theta$ on $\mathcal{L}$ such that $x_{i} \theta y_{i}$, for all $i \in I$, implies $\left(\bigvee_{i \in I} x_{i}\right) \theta\left(\bigvee_{i \in I} y_{i}\right)$ and $\left(\bigwedge_{i \in I} x_{i}\right) \theta\left(\bigwedge_{i \in I} y_{i}\right)$. Conversely, any equivalence relation $\theta$ on $\mathcal{L}$ satisfying these conditions is a complete congruence. Indeed, for such an equivalence relation $\theta$, the set of equivalence classes $\mathcal{L} / \theta$ is a complete lattice with the obvious order and the canonical map $h: \mathcal{L} \rightarrow \mathcal{L} / \theta$ is a complete homomorphism with kernel $\theta$.

The intersection of any collection of complete congruences is again a complete congruence. Hence the set of all complete congruences on $\mathcal{L}$ forms a complete lattice, with its meet operation being set intersection and its joins given by $\bigvee_{i \in I} \theta_{i}=\bigcap\left\{\psi: \theta_{i} \subseteq\right.$ $\psi$, for all $i \in I\}$.

If $\mathcal{L}$ is a complete lattice and $x \in \mathcal{L}$, define

$$
\begin{aligned}
& x_{-}=\bigvee\{y \in \mathcal{L}: x \not \leq y\} \text { and } \\
& x^{*}=\bigwedge\left\{s_{-}: s \in \mathcal{L} \text { and } s \not \leq x\right\} .
\end{aligned}
$$

Note that $x \leqslant x^{*}$, and that $x \leqslant y$ implies $x^{*} \leqslant y^{*}$. Of course, $x^{*} \leqslant\left(x^{*}\right)^{*}$, and this inequality may be strict. Let

$$
\mathcal{J}_{\mathcal{L}}=\{y \in \mathcal{L}: y \neq 0 \text { and } y-\neq 1\}
$$

so that

$$
x^{*}=\bigwedge\left\{s_{-}: s \in \partial_{\mathcal{L}} \text { and } s \not x\right\} .
$$

Finally, we observe that ${ }^{*}$ is a complete meet homomorphism: that is, $\left(\bigwedge x_{i}\right)^{*}=\bigwedge x_{i}^{*}$.

A complete lattice is completely distributive if it satisfies the identity

$$
\bigwedge_{i \in I} \bigvee_{j \in J} x_{i j}=\bigvee_{f \in J^{I}} \bigwedge_{i \in I} x_{i f(i)}
$$

where $J^{I}$ denotes the set of all $f: I \rightarrow J$. This identity is equivalent to its latticetheoretic dual (a good exercise). The importance of the * operation is in the following observation derived by the first author [7] from Raney [13].

LEMMA 1. A complete lattice $\mathcal{L}$ is completely distributive if and only if $x^{*}=x$, for all $x \in \mathcal{L}$.

There are three simple classes of examples of completely distributive lattices.

1. The lattice of all subsets of any set is completely distributive. 
2. Any complete chain (nest) is completely distributive.

3. Let $\mathcal{P}$ be any ordered set (poset). A subset $I \subseteq \mathcal{P}$ is an order ideal if $x \leqslant y \in I$ implies $x \in I$. The collection $\mathcal{O}(\mathcal{P})$ of all order ideals of $\mathcal{P}$ is closed under arbitrary set unions and intersections, and hence forms a completely distributive lattice. Indeed, a complete lattice $\mathcal{L}$ is completely distributive if and only if there is a complete surjective homomorphism $h: \mathcal{O}(\mathcal{P}) \rightarrow \mathcal{L}$, for some ordered set $\mathcal{P}[\mathbf{1 2}]$.

A nice survey of completely distributive lattices and their applications to operator theory may be found in Hopenwasser [5].

Now let $X$ be a (real or complex) Banach space. Let $\mathcal{B}(X)$ denote the algebra of bounded linear operators on $X$, and let $\mathrm{e}(X)$ denote the (complete) lattice of closed linear subspaces of $X$. (Throughout, our results apply to any topological vector space satisfying the Hahn-Banach Theorem: given a closed subspace $S$ and $x \notin S$, there exists a continuous linear functional $f$ such that $S \subseteq \operatorname{ker} f$ and $f(x)=1$. In this context, $\mathcal{B}(X)$ is the algebra of continuous linear operators on $X$.) For any set $R \subseteq \mathcal{B}(X)$, and for any set $S \subseteq \mathrm{e}(X)$, define

$$
\begin{aligned}
& \text { Alg } S=\{T \in \mathcal{B}(X): T(M) \subseteq M, \text { for all } M \in S\}, \\
& \text { Lat } R=\{M \in \mathcal{C}(X): T(M) \subseteq M, \text { for all } T \in R\} .
\end{aligned}
$$

Then $\operatorname{Alg} S$ is a subalgebra of $\mathcal{B}(X)$ and Lat $R$ is a complete $0-1$ sublattice of $\mathcal{C}(X)$. A sublattice $\mathcal{L} \subseteq \mathfrak{C}(X)$ is reflexive if and only if $\mathcal{L}=$ Lat $R$ for some $R \subseteq \mathcal{B}(X)$, or equivalently, $\mathcal{L}=\operatorname{Lat}$ Alg $\mathcal{L}$ (by standard results about Galois connections).

The crucial lemma from [7] (see Bandelt [2]) can be stated as follows.

Lemma 2. Let $X$ be a Banach space, and let $\mathcal{L}$ be a complete $0-1$ sublattice of $\mathfrak{C}(X)$. Let $R_{\mathcal{L}}$ be the set of rank-1 operators in Alg $\mathcal{L}$. Then, for $K \in \mathfrak{C}(X)$, we have $K \in$ Lat $R_{\mathcal{L}}$ if and only if there exists $N \in \mathcal{L}$ such that $N \subseteq K \subseteq N^{*}$ (with $N^{*}$ computed in $\mathcal{L})$.

\section{AN ABSTRACT VERSION OF Lat $R_{\mathcal{L}}$}

Now let $\mathcal{C}$ be a complete lattice, and let $\mathcal{L}$ be a complete 0-1 sublattice of $\mathcal{C}$. In view of Lemma 2, we define $\hat{\mathcal{L}}$ to be the set of all $k \in \mathcal{C}$ such that $n \leqslant k \leqslant n^{*}$, for some $n \in \mathcal{L}$ (with $n^{*}$ computed in $\mathcal{L}$ ). Clearly $\mathcal{L} \subseteq \widehat{\mathcal{L}}$. The main results in this section are proved in a more general setting in Bandelt [2]; our objective is to provide straightforward proofs involving only simple calculations.

LEMMA 3. The subset $\widehat{\mathcal{L}}$ is a complete $0-1$ sublattice of $\mathcal{C}$.

Proof: It is clear that $0,1 \in \widehat{\mathcal{L}}$. Let $k_{i} \in \widehat{\mathcal{L}}$, with say $n_{i} \leqslant k_{i} \leqslant n_{i}^{*}$ and $n_{i} \in \mathcal{L}$ $(i \in I)$. Then $\Lambda n_{i} \leqslant \Lambda k_{i} \leqslant \Lambda n_{i}^{*}=\left(\bigwedge n_{i}\right)^{*}$ and $\bigvee n_{i} \leqslant \bigvee k_{i} \leqslant \bigvee n_{i}^{*} \leqslant\left(\bigvee n_{i}\right)^{*}$, whence $\wedge k_{i}$ and $\bigvee k_{i}$ are in $\widehat{\mathcal{L}}$. 
We can simplify the computation of $\widehat{\mathcal{L}}$ as follows. For $x \in \mathcal{C}$, define $m(x)=\bigvee\{j \in$ $\left.\partial_{\mathcal{L}}: j \leqslant x\right\}$, so that $j \leqslant x$ if and only if $j \leqslant m(x)$ holds for $j \in \partial_{\mathcal{L}}$.

LEMMA 4. For $k \in \mathfrak{C}$, we have $k \in \widehat{\mathcal{L}}$ if and only if $k \leqslant m(k)^{*}$.

Proof: As $m(k) \leqslant k$, if $k \leqslant m(k)^{*}$ then $k \in \hat{\mathcal{L}}$.

Conversely, note that for $x \in \mathcal{L}$ we have

$$
\begin{aligned}
x^{*} & =\bigwedge\left\{s_{-}: s \in \partial_{\mathcal{L}} \text { and } s \not \leq x\right\} \\
& =\bigwedge\left\{s_{-}: s \in \partial_{\mathcal{L}} \text { and } s \not \leq m(x)\right\} \\
& =m(x)^{*} .
\end{aligned}
$$

So if $k \in \widehat{\mathcal{L}}$ with say $n \leqslant k \leqslant n^{*}$ and $n \in \mathcal{L}$, then $k \leqslant n^{*}=m(n)^{*} \leqslant m(k)^{*}$ as desired. $\square$ REMARK. Now in general, for $k \in \widehat{\mathcal{L}}, m(k)$ need not be the least element $n$ of $\mathcal{L}$ such that $n \leqslant k \leqslant n^{*}$. The description of the maximal intervals of the form $\left[x, x^{*}\right]$ in $\mathcal{L}$ is given in Bandelt [1]. (These intervals may overlap.) For the sake of completeness, we give a quick synopsis of the most important part of Bandelt's analysis.

Define $x_{+}$and $x_{*}$ dually to $x_{-}$and $x^{*}$, respectively. Thus

$$
\begin{aligned}
& x_{+}=\bigwedge\{y \in \mathcal{L}: x \nsupseteq y\} \text { and } \\
& x_{*}=\bigvee\left\{s_{+}: s \in \mathcal{L} \text { and } s \nsupseteq x\right\} .
\end{aligned}
$$

Note that $x \geqslant x_{*}$, and that $x \leqslant y$ implies $x_{*} \leqslant y_{*}$.

ThEOREM 5. Let $\mathcal{L}$ be a complete lattice, and let $x, y \in \mathcal{L}$. Then

1. $\left(x^{*}\right)_{*} \leqslant x$,

2. $\left(\left(x^{*}\right)_{*}\right)^{*}=x^{*}$,

3. $y^{*} \geqslant x^{*}$ if and only if $y \geqslant\left(x^{*}\right)_{*}$.

Hence $\left(x^{*}\right)_{*}$ is the least element $y \in \mathcal{L}$ such that $y^{*}=x^{*}$.

Proof: First, note that $s_{+-} \leqslant s$, for all $s \in \mathcal{L}$. For $r \not s_{+}$implies $r \leqslant s$, and hence $s_{+-}=\bigvee\left\{r: r \not s_{+}\right\} \leqslant s$.

To prove (1), since $\left(x^{*}\right)_{*}=\bigvee\left\{s_{+}: s \nsupseteq x^{*}\right\}$, we need to know that $s \nsupseteq x^{*}$ implies $s_{+} \leqslant x$. But $s_{+} \not \leq x$ implies $s_{+-} \geqslant x^{*}$, and hence by the observation above $s \geqslant x^{*}$.

It follows from (1) that $\left(\left(x^{*}\right)_{*}\right)^{*} \leqslant x^{*}$, while the dual of (1) applied to $x^{*}$ yields $\left(\left(x^{*}\right)_{*}\right)^{*} \geqslant x^{*}$. This proves (2), and (3) follows easily from (1) and (2).

Now let us show that $\widehat{\hat{L}}=\widehat{\mathcal{L}}$. For this purpose, let $x_{\Theta}$ and $x^{\oplus}$ be the corresponding operations to $x_{-}$and $x^{*}$, defined in $\hat{\mathcal{L}}$.

LеммA 6. If $x \in \mathcal{L}$, then $x_{\Theta}=x_{-}$. Thus $\partial_{\mathcal{L}} \subseteq \partial_{\widehat{\mathcal{L}}}$.

Proof: Clearly $x_{-}=\bigvee\{y \in \mathcal{L}: x \not \leq y\} \leqslant \bigvee\{y \in \widehat{\mathcal{L}}: x \not \leq y\}=x_{\ominus}$. For the reverse inclusion, first note that, by convention $0_{\Theta}=0_{-}=0$ and that, if $0 \neq x \notin \partial_{\mathcal{L}}$ then $x_{-}=$ 
$x_{\Theta}=1$. Thus without loss of generality we may assume that $x \in \partial_{\mathcal{L}}$. Then for $y \in \widehat{\mathcal{L}}$, $x \not \leq y$ implies $x \not \leq m(y)$, and hence $x_{-} \geqslant m(y)^{*} \geqslant y$. Thus $x_{-} \geqslant \bigvee\{y \in \widehat{\mathcal{L}}: x \not y\}=x_{\Theta}$, whence we obtain equality.

This yields a straightforward proof of a nice theorem from Bandelt [2].

THEOREM 7. Let $\mathcal{L}$ be a complete $0-1$ sublattice of a complete lattice $\mathcal{C}$. Then $\widehat{\hat{\mathcal{L}}}=\hat{\mathcal{L}}$.

Proof: First we note, using Lemma 6, that if $k \in \widehat{\mathcal{L}}$,

$$
\begin{aligned}
k^{\oplus} & =\bigwedge\left\{s_{\Theta}: s \in \partial_{\widehat{L}} \text { and } s \not k\right\} \\
& \leqslant \bigwedge\left\{s_{\ominus}: s \in \partial_{\mathcal{L}} \text { and } s \not k k\right\} \\
& =\bigwedge\left\{s_{-}: s \in \mathcal{J}_{\mathcal{L}} \text { and } s \not \leq m(k)\right\} \\
& =m(k)^{*} .
\end{aligned}
$$

Now suppose $t \in \mathcal{C}, k \in \widehat{\mathcal{L}}$ and $k \leqslant t \leqslant k^{\oplus}$. Then $m(k) \leqslant k \leqslant t \leqslant k^{\oplus} \leqslant m(k)^{*}$, so that $t \in \widehat{\mathcal{L}}$. Thus $\widehat{\hat{\mathcal{L}}} \subseteq \widehat{\mathcal{L}}$, and the reverse inclusion is obvious.

There is also a simple characterisation of $\partial_{\hat{L}}$.

THEOREM 8. Let $0 \neq k \in \widehat{\mathcal{L}}$. Then $k \in \mathcal{J}_{\overline{\mathcal{L}}}$ if and only if there exists $j \in \partial_{\mathcal{L}}$ such that $k \leqslant j$.

Proof: If $k \leqslant j \in \mathcal{J}_{\mathcal{L}}$ then $k_{\ominus} \leqslant j_{\ominus}=j_{-}<1$, whence $k \in \partial_{\hat{\mathcal{L}}}$.

Conversely, suppose $k_{\ominus}<1$. Let $d=\bigwedge\{x \in \mathcal{L}: k \leqslant x\}$, so that $k \leqslant x$ if and only if $d \leqslant x$ for $x \in \mathcal{L}$. Then

$$
d_{-}=\bigvee\{x \in \mathcal{L}: d \not \leq x\} \leqslant \bigvee\{x \in \mathcal{L}: k \not \leq x\} \leqslant k_{\ominus}<1
$$

whence $d \in \partial_{\mathcal{L}}$.

In view of the previous considerations, we would like to find lattices in which the intervals $\left[m(x), x^{*}\right]$ fit together nicely. This leads us indirectly to the next problem.

\section{Finding THE MINIMUM COMPLETELY DISTRIBUTIVE CONGRUENCE}

There is a least complete congruence $\Delta$ on a complete lattice $\mathcal{L}$ such that $\mathcal{L} / \Delta$ is completely distributive. Indeed, $\Delta$ is just the intersection of all those complete congruences $\theta$ on $\mathcal{L}$ such that $\left(\bigwedge_{i \in I} \bigvee_{j \in J} x_{i j}\right) \theta\left(\bigvee_{f \in J I} \bigwedge_{i \in I} x_{i f(i)}\right)$ always holds. In this section we want to find a description of $\Delta$ in terms of the operation *.

If $\theta$ is any complete congruence on $\mathcal{L}$, then each $\theta$-class has a greatest element and a least element. The greatest element of the $\theta$-class containing $x$ is denoted by $\alpha_{\theta}(x)$, and the least element by $\beta_{\theta}(x)$. Our first observation is just the complete lattice version of a familiar result for finite lattices (see, for example, [3]). 
Lemma 9. Let $\mathcal{L}$ be a complete lattice, and let $f: \mathcal{L} \rightarrow \mathcal{L}$. Then $f=\alpha_{\theta}$ for some complete congruence $\theta$ if and only if

1. $x \leqslant f(x)=f(f(x))$, for all $x \in \mathcal{L}$, and

2. $f$ preserves arbitrary meets, that is, $f\left(\bigwedge_{i \in I} x_{i}\right)=\bigwedge_{i \in I} f\left(x_{i}\right)$.

Indeed, if conditions (1) and (2) of the lemma hold, the range $f(\mathcal{L})$ of $f$ is a complete meet subsemilattice of $\mathcal{L}$ containing 1 , and hence a complete lattice with the order inherited from $\mathcal{L}$. Moreover, $f$ is a complete homomorphism onto $f(\mathcal{L})$. The critical observation is that, because $x \leqslant f(y)$ if and only if $f(x) \leqslant f(y), f\left(\bigvee_{i \in I} x_{i}\right)$ is the least upper bound in $f(\mathcal{L})$ of $\left\{f\left(x_{i}\right): i \in I\right\}$.

Lemma 10. Let $\mathcal{L}$ be a complete lattice, and let $\eta$ be a complete congruence on $\mathcal{L}$. Then the following are equivalent.

1. $\mathcal{L} / \eta$ is completely distributive.

2. $x^{*} \eta x$, for all $x \in \mathcal{L}$.

3. $u^{*}=u$ whenever $u=\alpha_{\eta}(x)$, for some $x \in \mathcal{L}$.

Proof: Given a complete congruence $\eta$ on $\mathcal{L}$, let $h: \mathcal{L} \rightarrow \mathcal{L} / \eta$ be the canonical homomorphism with $\eta=\operatorname{ker} h$. For $x \in \mathcal{L}$, let $x_{L}=\beta_{\eta}(x)$ and let $x_{U}=\alpha_{\eta}(x)$. Note that $h(x) \leqslant h(y)$ if and only if $x_{L} \leqslant y$ if and only if $x \leqslant y_{U}$ if and only if $x_{L} \leqslant y_{U}$.

Thus we calculate

$$
\begin{aligned}
h\left(z_{L-}\right) & =h\left(\bigvee\left\{s: z_{L} \not \leq s\right\}\right) \\
& =\bigvee\left\{h(s): h\left(z_{L}\right) \not \leq h(s)\right\} \\
& =h\left(z_{L}\right)_{-} \\
& =h(z)_{-} .
\end{aligned}
$$

Likewise,

$$
\begin{aligned}
h(x)^{*} & =\bigwedge\left\{h(z)_{-}: h(z) \not h(x)\right\} \\
& =\bigwedge\left\{h\left(z_{L}\right)_{-}: z_{L} \not x_{U}\right\} \\
& =\bigwedge\left\{h\left(z_{L_{-}}\right): z_{L} \not \leq x_{U}\right\} \\
& =h\left(\bigwedge\left\{z_{L-}: z_{L} \not \leq x_{U}\right\}\right) \\
& =h\left(\bigwedge\left\{z_{-}: z \not \leq x_{U}\right\}\right) \\
& =h\left(\left(x_{U}\right)^{*}\right),
\end{aligned}
$$

where for the fifth equality we have used $z\left\lfloor x_{U}\right.$ if and only if $z_{L} \leq x_{U}$ and $z_{L-} \leqslant z_{-}$. 
So, if $\mathcal{L} / \eta$ is completely distributive, then

$$
h(x)=h(x)^{*}=h\left(\left(x_{U}\right)^{*}\right) \geqslant h\left(x^{*}\right) \geqslant h(x),
$$

for all $x \in \mathcal{L}$. Thus (1) implies (2). Clearly (2) implies (3). Finally, if (3) holds, then

$$
h(x)^{*}=h\left(\left(x_{U}\right)^{*}\right)=h\left(x_{U}\right)=h(x),
$$

for all $x \in \mathcal{L}$, whence $\mathcal{L} / \eta$ is completely distributive.

COROLLARY 11. If $\mathcal{L}$ is a complete lattice, the least complete congruence $\Delta$ on $\mathcal{L}$ such that $\mathcal{L} / \Delta$ is completely distributive is the smallest complete congruence containing $\left\langle x, x^{*}\right\rangle$, for all $x \in \mathcal{L}$.

Note that if $\theta$ and $\varphi$ are complete congruences with $\theta \subseteq \varphi$, then $\alpha_{\theta}(x) \leqslant \alpha_{\varphi}(x)$, for all $x \in \mathcal{L}$. Combining this observation with the previous two lemmas, we obtain our first description of $\alpha_{\Delta}$ (and hence implicitly of $\Delta$, since $\Delta$ is the kernel of the map $\left.x \mapsto \alpha_{\Delta}(x)\right)$.

THEOREM 12. Let $\mathcal{L}$ be a complete lattice, and let $\Delta$ be the least complete congruence on $\mathcal{L}$ such that $\mathcal{L} / \Delta$ is completely distributive. Then $\alpha_{\Delta}$ is the least (pointwise) map $f: \mathcal{L} \rightarrow \mathcal{L}$ satisfying

1. $x \leqslant f(x)=f(f(x))$, for all $x \in \mathcal{L}$,

2. $f$ preserves arbitrary meets, that is, $f\left(\bigwedge_{i \in I} x_{i}\right)=\bigwedge_{i \in I} f\left(x_{i}\right)$,

3. $f(x)^{*}=f(x)$, for every $x \in \mathcal{L}$.

Now we can actually construct the map $f=\alpha_{\Delta}$ of the preceding theorem as follows. Let $\mathcal{F}=\left\{u \in \mathcal{L}: u^{*}=u\right\}$. Then $\mathcal{F}$ is a complete meet subsemilattice of $\mathcal{L}$ containing 1 . This makes $\mathcal{F}$ a complete lattice also, with its meet operation $\Lambda$ inherited from $\mathcal{L}$ and joins given by $\sum X=\bigwedge\{u \in \mathcal{F}: u \geqslant \bigvee X\}$. Note that (3) gives $f(\mathcal{L}) \subseteq \mathcal{F}$.

For $x \in \mathcal{L}$, define $\bar{x}=\bigwedge\{u \in \mathcal{F}: u \geqslant x\}$. Thus $\bar{x}$ is the least element $y \in \mathcal{L}$ such that $y \geqslant x$ and $y^{*}=y$. In particular, the map $f_{0}: x \mapsto \bar{x}$ satisfies properties (1) and (3) of Theorem 12. However, it may not preserve infinite meets (see below).

The element $\bar{x}$ can be described inductively as follows. Let $x^{0}=x$. For successor ordinals define $x^{\gamma+1}=\left(x^{\gamma}\right)^{*}$; for limit ordinals, $x^{\lambda}=\bigvee\left\{x^{\gamma}: \gamma<\lambda\right\}$. This sequence eventually stabilises at some value $\widetilde{x}$ with $\widetilde{x}^{*}=\widetilde{x}$. If $u \in \mathcal{F}$ and $u \geqslant x$, then inductively $u \geqslant x^{\gamma}$ for all $\gamma$, and hence $u \geqslant \tilde{x}$. Thus $\tilde{x}=\bar{x}$.

Now we proceed to construct the smallest meet-preserving map $f$ greater than or equal to $f_{0}$ in the traditional manner. Starting with $f_{0}(x)=\bar{x}$, for successor ordinals define $f_{\gamma+1}(x)=\sum\left\{\bigwedge_{y \in Y} f_{\gamma}(y): \bigwedge Y \leqslant x\right\}$, and for limit ordinals $f_{\lambda}(x)=\sum_{\beta} f_{\mathcal{\beta}}(x)$ This eventually stabilises in a map $f$ which satisfies (1)-(3). Moreover, it is the least possible such map, so $f=\alpha_{\Delta}$, as desired. 
REMARK. Interestingly, the map $f_{0}$ with $f_{0}(x)=\bar{x}$ preserves finite meets and infinite joins. The argument that $f_{0}$ preserves finite meets uses a straightforward induction to show that, for all $\gamma$,

$$
\bigwedge_{i \leqslant n} x_{i}^{\gamma} \leqslant\left(\bigwedge_{i \leqslant n} x_{i}\right)^{\gamma+1} .
$$

Thus if $\mathcal{L}$ satisfies the descending chain condition, then there are no proper infinite meets in $\mathcal{L}$, and $f_{0}$ preserves meets. So when $\mathcal{L}$ satisfies the DCC, we have $f_{0}=\alpha_{\Delta}$.

The example in Figure 1(a) shows that, even with the ACC, $f_{0}$ need not preserve infinite meets. In the example, $0^{*}=0,1^{*}=1$ and $x_{i+1}^{*}=x_{i}$, for all $i$. Hence $\overline{0}=0$ but $\bar{x}=1$, for all $x>0$, so that $f_{0}\left(\bigwedge x_{i}\right)=0$ while $\bigwedge f_{0}\left(x_{i}\right)=1$.

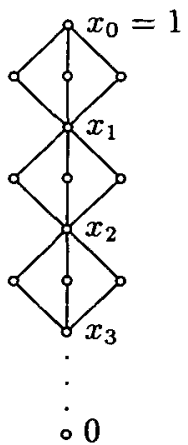

(a)

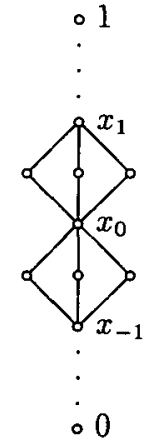

(b)

Figure 1

\section{G-LATtices}

In this section we shall investigate a class of complete lattices for which the map $\mathcal{L} \mapsto \widehat{\mathcal{L}}$ and the congruence $\Delta$ are well-behaved.

Recall that an element $p \in \mathcal{L}$ is completely join prime if $p \not \leq p_{-}$. Let $\mathcal{P}_{\mathcal{L}}$ denote the set of completely join prime elements of $\mathcal{L}$. Note that, by convention, $0_{-}=\bigvee \emptyset=0$ so $0 \notin \mathcal{P}_{\mathcal{L}}$. Clearly $\mathcal{P}_{\mathcal{L}} \subseteq \mathcal{J}_{\mathcal{L}}$, and this inclusion may be proper.

We say that $\mathcal{L}$ is a $\mathcal{G}$-lattice if every element of $\partial_{\mathcal{L}}$ is a join of completely join prime elements, that is, if $s \in \mathcal{J}_{\mathcal{L}}$ then $s=\bigvee\left\{p \in \mathcal{P}_{\mathcal{L}}: p \leqslant s\right\}$.

LEMMA 13. Let $\mathcal{L}$ be a $\mathcal{G}$-lattice. If $s \in \mathcal{J}_{\mathcal{L}}$ and $x \in \mathcal{L}$, then $s \leqslant x^{*}$ if and only if $s \leqslant x$.

Proof: If $p \in \mathcal{P}_{\mathcal{L}}$ and $p \not x$, then $x^{*} \leqslant p_{-}$and hence $p \not x^{*}$. Thus $p \leqslant x^{*}$ if and only if $p \leqslant x$. For $s \in \partial_{\mathcal{L}}, s \leqslant z$ if and only if $p \leqslant z$, for all $p \in \mathcal{P}_{\mathcal{L}}$ with $p \leqslant s$, whence the statement follows.

In the following, $m(x)$ is defined as before, namely $m(x)=\bigvee\left\{j \in \partial_{\mathcal{L}}: j \leqslant x\right\}$. 
TheOREM 14. If $\mathcal{L}$ is a $\mathcal{G}$-lattice, then for all $x \in \mathcal{L}$,

1. $x^{*}=x^{* *}=m(x)^{*}$,

2. $m(x)=x_{*}=\left(x^{*}\right)_{*}$.

Hence $\alpha_{\Delta}(x)=x^{*}$ and $\beta_{\Delta}(x)=m(x)$, and $\mathcal{L} / \Delta$ is isomorphic to the lattice of order ideals of $\mathcal{P}_{\mathcal{L}}$.

Proof: Lemma 13 and the definition of ${ }^{*}$ give $x^{*}=x^{* *}$, while $x^{*}=m(x)^{*}$ follows as in the proof of Lemma 4. Clearly $x_{*} \leqslant\left(x^{*}\right)_{*}$, and $\left(x^{*}\right)_{*} \leqslant m(x)$ by Theorem 5 . It remains to show that $m(x) \leqslant x_{*}$. Now $m(x)$ in a G-lattice is the join of the completely join prime elements less than or equal to $x$. If $p \in \mathcal{P}_{\mathcal{L}}$ and $p \leqslant x$, then $p_{-} \nsupseteq x$, whence $p=p_{-+} \leqslant \bigvee\left\{s_{+}: s \nsupseteq x\right\}=x_{*}$, as desired.

Since the map $x \mapsto x^{*}$ preserves arbitrary meets and $x^{* *}=x^{*}$, it follows from Theorem 12 that $x^{*}=\alpha_{\Delta}(x)$, for all $x$. Then $\beta_{\Delta}(x)$ is the least element $y$ with $y^{*}=x^{*}$. By Theorem 5 , this is $\left(x^{*}\right)_{*}$, which equals $m(x)$ by (2).

Finally, $\mathcal{L} / \Delta$ is order-isomorphic to $\left\{\beta_{\Delta}(x): x \in \mathcal{L}\right\}=\{m(x): x \in \mathcal{L}\}$. This latter is easily seen to be isomorphic to the lattice of order ideals of $\mathcal{P}_{\mathcal{L}}$.

Thus the blocks $\left[m(x), x^{*}\right]$ which are "filled in" in going from $\mathcal{L}$ to $\widehat{\mathcal{L}}$ are disjoint for $\mathcal{G}$-lattices (since they correspond to $\Delta$-classes). Likewise, the description of $\Delta$ is particularly simple for G-lattices: $x \Delta y$ if and only if $x^{*}=y^{*}$ if and only if $m(x)=m(y)$.

The following observation allows us to find many examples of $\mathcal{G}$-lattices, two of which are given in Figure 2.

Lемма 15. Let $\mathcal{L}$ be a complete lattice with $\bigvee \mathcal{P}_{\mathcal{L}}=1$. The following are equivalent.

1. $\mathcal{L}$ is a $\mathcal{G}$-lattice.

2. If $x \leqslant p \in \mathcal{P}_{\mathcal{L}}$, then $x$ is a join of completely join prime elements.

If, additionally, $\mathcal{L}$ is finite, these conditions are equivalent to

3. If $x$ is join irreducible and $0 \neq x \leqslant p \in \mathcal{P}_{\mathcal{L}}$, then $x \in \mathcal{P}_{\mathcal{L}}$.

Proof: Since $\mathcal{P}_{\mathcal{L}} \subseteq \partial_{\mathcal{L}}$ and $0 \neq x \leqslant y \in \partial_{\mathcal{L}}$ implies $x \in \partial_{\mathcal{L}},(1)$ implies (2). On the other hand, suppose that (2) holds and let $x \in \partial_{\mathcal{L}}$. Then $x_{-}<1$ implies that $x \leqslant p$, for some $p \in \mathcal{P}_{\mathcal{L}}$, whence $x$ is a join of elements in $\mathcal{P}_{\mathcal{L}}$. Thus $\mathcal{L}$ is a $\mathcal{G}$-lattice. Condition (3) is clearly equivalent to (2) for finite lattices, but easier to check.

The following observations hold in any lattice, but are particularly relevant in $\mathcal{G}$ lattices.

Leмma 16. Let $\mathcal{L}$ be a complete lattice. Then

1. $x<1$ implies $x^{*}<1$ if and only if $\vee \partial_{\mathcal{L}}=1$,

2. $0^{*}=0$ if and only if $\bigwedge\left\{s_{-}: s \in \partial_{\mathcal{L}}\right\}=0$.

Proof: (1) follows from the fact that $x^{*}<1$ if and only if $j \not \leq x$, for some $j \in \partial_{\mathcal{L}}$. (2) is immediate because $0^{*}=\bigwedge\left\{s_{-}: s \in \partial_{\mathcal{L}}\right\}$. 


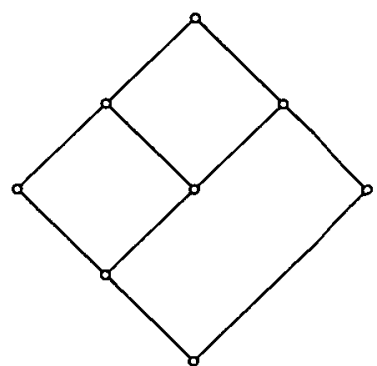

(a)

Figure 2

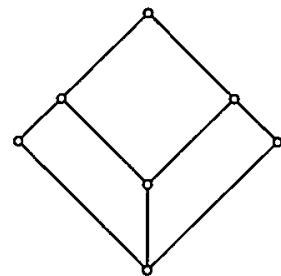

(b)

This brings us to an interesting notion. If $\mathcal{L}$ and $\mathcal{K}$ are complete lattices, let us say that $\mathcal{L}$ collapses to $\mathcal{K}$ if there is a complete surjective homomorphism $h: \mathcal{L} \rightarrow \mathcal{K}$ such that $h^{-1}(0)=\{0\}$ and $h^{-1}(1)=\{1\}$. Thus $\mathcal{L}$ collapses to a completely distributive lattice if and only if $x \Delta 0$ implies $x=0$ and $y \Delta 1$ implies $y=1$, equivalently, $\alpha_{\Delta}(0)=0$ and $\beta_{\Delta}(1)=1$. As an immediate consequence of the preceding lemma, and the fact that $x^{*}=\alpha_{\Delta}(x)$ in a $\mathcal{G}$-lattice, we have the following result.

THEOREM 17. The following are equivalent for a G-lattice.

1. $\mathcal{L}$ collapses to a completely distributive lattice.

2. $\vee \partial_{\mathcal{L}}=1$ and $\bigwedge\left\{s_{-}: s \in \partial_{\mathcal{L}}\right\}=0$.

3. $\bigvee \mathcal{P}_{\mathcal{L}}=1$ and $\bigwedge\left\{p_{-}: p \in \mathcal{P}_{\mathcal{L}}\right\}=0$.

For example, the lattice in Figure 2(a) collapses to a distributive lattice, while that in Figure 2(b) does not. The example of Figure 1(b) shows that, in general, (2) is not sufficient to imply (1). (Clearly (1) implies (2).)

The three equivalences of Theorem 17 also hold for finite lattices, but for slightly different reasons. Recall that $x^{0}=x$ and $x^{n+1}=\left(x^{n}\right)^{*}$. In a finite lattice $\mathcal{L}$, there is an integer $n$ such that $x^{n+1}=x^{n}$, for all $x \in \mathcal{L}$. The mapping $f: \mathcal{L} \rightarrow \mathcal{L}$ given by $f(x)=x^{n}$ preserves meets (by induction because $x \mapsto x^{*}$ does), and hence by Theorem 12 , $f(x)=\alpha_{\Delta}(x)$. Applying Lemma 16, we see that conditions (1) and (2) of Theorem 17 are equivalent for finite lattices.

On the other hand, if $\mathcal{L}$ is finite then $\beta_{\Delta}(x)=\bigvee\left\{p \in \mathcal{P}_{\mathcal{L}}: p \leqslant x\right\}$, for all $x \in \mathcal{L}$. It follows that $\beta_{\Delta}(1)=1$ if and only if $\bigvee \mathcal{P}_{\mathcal{L}}=1$. Since the completely meet prime elements of $\mathcal{L}$ are precisely those of the form $p_{-}$, for some $p \in \mathcal{P}_{\mathcal{L}}$, dually we have $\alpha_{\Delta}(0)=0$ if and only if $\bigwedge\left\{p_{-}: p \in \mathcal{P}_{\mathcal{L}}\right\}=0$. Thus conditions (1) and (3) are equivalent for finite lattices.

Note that a complete lattice $\mathcal{L}$ collapses to a finite chain (nest) if and only if 1 is completely join irreducible and 0 is completely meet irreducible. It would be interesting to find necessary and sufficient conditions for a complete lattice to collapse to a chain.

Recall that a lattice is (upper) semimodular if, whenever $a$ and $c$ are incomparable, 
$a>b>a \wedge c$ implies that there exists $d$ such that $a \wedge c<d \leqslant c$ and $a \wedge(b \vee d)=b$. (This is the definition used for general lattices; there are other equivalent versions for lattices containing no infinite chain. If a semimodular lattice $\mathcal{L}$ has a finite maximal chain, then every maximal chain of $\mathcal{L}$ has the same length, which is called the length of $\mathcal{L}$. Recall that $x \succ y$ if $x>y$ and there is no element $z$ such that $x>z>y$. The notation $x \succeq y$ means $x \succ y$ or $x=y$. In the next theorem, we use the fact that a lattice with no infinite chain is semimodular if and only if $x \succ y$ implies $x \vee z \succeq y \vee z$.)

THEOREM 18. If $\mathcal{L}$ is a finite-length semimodular $\mathcal{S}$-lattice with $\bigvee \mathcal{P}_{\mathcal{L}}=1$, then $\mathcal{L}$ is finite and (completely) distributive.

Proof: It is not hard to see that a lattice with no infinite chain can only have finitely many completely join prime elements. With this observation, it suffices to show that every element of $\mathcal{L}$ is a join of completely join prime elements. For $p \in \mathcal{P}_{\mathcal{L}}$, let $p_{\dagger}$ denote the unique element covered by $p$, that is, $p_{\dagger}=\bigvee\{t \in \mathcal{L}: t<p\}$.

Let $x$ be a nonzero element of $\mathcal{L}$. As $x \leqslant \vee \mathcal{P}_{\mathcal{L}}=1$, we can find a minimal order ideal $I$ of $\mathcal{P}_{\mathcal{L}}$ such that $\bigvee I \geqslant x$. By Lemma 15 , each $p_{\uparrow}$ is a (possibly empty) join of elements of $\mathcal{P}_{\mathcal{L}}$, and hence $x \not \leq p_{\dagger} \vee \bigvee(I \backslash\{p\})$, for all $p \in I$. Let $p \in I$. Then by semimodularity, $\bigvee I \succ p_{\dagger} \vee \bigvee(I \backslash\{p\}) \nsucceq x$. But this implies that $x \vee p_{\uparrow} \vee \bigvee(I \backslash\{p\})=\bigvee I \geqslant p$. Since $p$ is join prime, this implies $p \leqslant x$. Thus $p \leqslant x$, for all $p \in I$, whence $\bigvee I \leqslant x$. So $\bigvee I=x$, and we have shown that every element of $\mathcal{L}$ is a join of completely join prime elements, whence $\mathcal{L}$ is isomorphic to the lattice of order ideals of $\mathcal{P}_{\mathcal{L}}$.

Figure 3 gives an example of an infinite distributive $\mathcal{G}$-lattice with $\bigvee \mathcal{P}_{\mathcal{L}}=1$ and $\wedge\left\{p_{-}: p \in \mathcal{P}_{\mathcal{L}}\right\}=0$, and satisfying the DCC, but which is still not completely distributive.

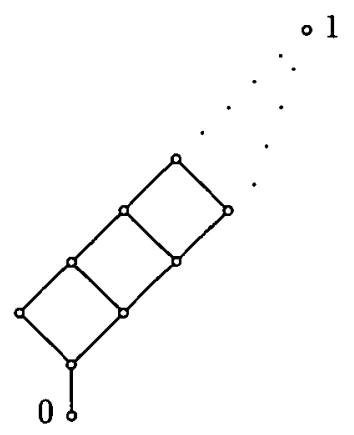

Figure 3

\section{J-LATTICES}

In this section we turn our attention to a particularly nice class of complete lattices, those which collapse to a complete atomic Boolean algebra. 
To simplify notation, for a complete lattice $\mathcal{L}$, let $\mathcal{J}=\partial_{\mathcal{L}}$ and

$$
\partial_{-}=\left\{a_{-}: a \in \partial\right\}
$$

Following $[\mathbf{1 1}]$, we say that $\mathcal{L}$ is a $\mathcal{J}$-lattice if

$$
\begin{aligned}
& \text { 1. } \vee \mathcal{J}=1, \\
& \text { 2. } \wedge \partial_{-}=0, \\
& \text { 3. } a \vee a_{-}=1, \text { for all } a \in J, \\
& \text { 4. } a \wedge a_{-}=0, \text { for all } a \in J .
\end{aligned}
$$

As observed in [9], there are redundancies in the above four conditions. For example, conditions (1) and (2) together with either (3) or (4) implies all four. We note that lattices satisfying condition (2) are called "nice" lattices in [6]. For some other results related to these conditions see $[\mathbf{8}],[\mathbf{1 1}]$.

Every complete atomic Boolean algebra is a $\partial$-lattice. Indeed, for such lattices $\partial$ is the set of atoms and, for every atom $a, a_{-}$is the Boolean complement of $a$ [7]. Figure 4 gives two other examples of $\partial$-lattices, where the one on the right is called the pentagon, and the structural characterisation of Theorem 22 below indicates how we can construct many more.
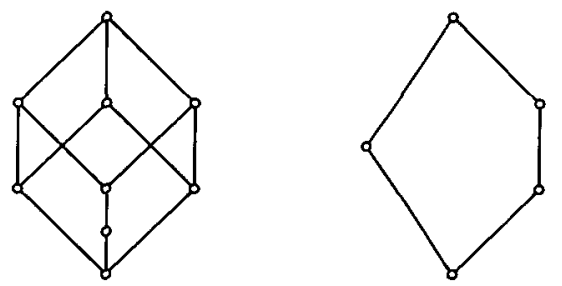

Figure 4

Let us begin by proving some elementary facts about $\mathcal{J}$-lattices.

Lemma 19. Let $\mathcal{L}$ be a $\mathcal{J}$-lattice. Then

1. $\mathcal{J}=\mathcal{P}_{\mathcal{L}}$, and thus $\mathcal{L}$ is a $\mathcal{G}$-lattice,

2. every $a \in \mathcal{J}$ is an atom,

3. every nonzero element of $\mathcal{L}$ is greater than or equal to some element of $\mathcal{J}$.

Proof: If $a \in \mathcal{J}$, then $a \neq 0$ and $a \wedge a_{-}=0$, whence $a \not a_{-}$. Thus $a \in \mathcal{P}_{\mathcal{L}}$. If $0 \leqslant x<a$ and $a \in \partial$, then $x \leqslant a \wedge a_{-}=0$, so $a$ is an atom. Likewise, if $x>0=\wedge \partial_{-}$, then $x \not a_{-}$, for some $a \in \mathcal{J}$, whence $a \leqslant x$.

The lattices in Figures 2 and 3 are $\mathcal{G}$-lattices which are not $\partial$-lattices. The threeelement chain is an even easier example. 
Now in any complete lattice an element $x$ is completely meet prime if $x \nsupseteq x_{+}=$ $\bigwedge\{y \in \mathcal{L}: y \not \leq x\}$. Since $\bigwedge \emptyset=1$, the element 1 is not completely meet prime. Completely join prime elements and completely meet prime elements occur in pairs: $x$ is

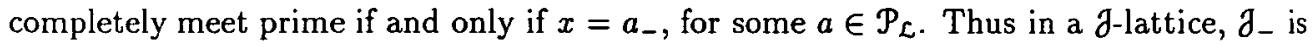
the set of completely meet prime elements of $\mathcal{L}$.

Lemma 20. The complete lattice $\mathcal{L}$ is a $\partial$-lattice if and only if

1. $\partial$ is an antichain,

2. $\bigvee \mathfrak{d}=1$,

3. $\wedge \partial_{-}=0$.

Proof: Suppose $\mathcal{L}$ is a $\mathcal{J}$-lattice. By Lemma $19(2)$, every element of $\mathcal{J}$ is an atom of $\mathcal{L}$. Thus $\mathcal{J}$ is an antichain, and $\mathcal{L}$ satisfies (1)-(3).

Conversely, assume that $\mathcal{L}$ satisfies (1)-(3). If $a$ and $b$ are distinct elements of $\partial$, then $b \leqslant a_{-}$, whence $a \vee a_{-} \geqslant \bigvee \mathcal{J}=1$ and $b \wedge b_{-} \leqslant \wedge \partial_{-}=0$. Therefore $\mathcal{L}$ is a $\partial$-lattice.

THEOREM 21. If $\mathcal{L}$ is a $\partial$-lattice, then so is $\widehat{\mathcal{L}}$.

Proof: Let $\mathcal{L}$ be a $\mathcal{J}$-lattice. Then $0^{*}=\wedge \mathcal{J}_{-}=0$, and hence the atoms of $\mathcal{L}$ are also atoms of $\widehat{\mathcal{L}}$. By Theorem 8 , we conclude that $\partial_{\widehat{L}}=\mathcal{J}$. Thus $\widehat{\mathcal{L}}$ satisfies conditions (1) and (2) of Lemma 20. By Lemma $6, a_{\ominus}=a_{-}$, for each $a \in \mathcal{J}$, so $\widehat{\mathcal{L}}$ also satisfies (3). Thus $\hat{\mathcal{L}}$ is a $\mathrm{d}$-lattice.

Now we can give a characterisation of $\partial$-lattices.

THEOREM 22. A complete lattice $\mathcal{L}$ is a $\mathcal{J}$-lattice if and only if it collapses to a complete atomic Boolean algebra, that is, if and only if there exists a complete atomic Boolean algebra $\mathcal{B}$ and a complete surjective homomorphism $h: \mathcal{L} \rightarrow \mathcal{B}$ such that $h^{-1}(0)=\{0\}$ and $h^{-1}(1)=\{1\}$.

Proof: Given a $\mathcal{J}$-lattice $\mathcal{L}$, we can apply Theorem 14 and Lemma 16 to see that $\mathcal{L} / \Delta \cong \mathfrak{P}(\mathcal{\partial})$, where $\mathfrak{P}(\mathcal{\partial})$ denotes the lattice of subsets of $\mathcal{J}$, or just directly check that $h: \mathcal{L} \rightarrow \mathfrak{P}(\mathfrak{\partial})$ given by $h(x)=\{a \in \mathfrak{J}: a \leqslant x\}$ is a collapsing homomorphism.

Conversely, assume that there exist such an $h$ and $\mathcal{B}$. Let $\mathcal{K}$ denote the set of least preimages of atoms of $\mathcal{B}$, which exist because $h$ is complete and onto. Let us show that $\mathcal{K}=\mathscr{J}$ in $\mathcal{L}$. If $a \in \mathcal{K}$, then $a \neq 0$ and $a_{-}$is the largest preimage of the complementary coatom to $h(a)$ in $\mathcal{B}$ (since $h(x) \geqslant h(a)$ if and only if $x \geqslant a$ by the minimality of $a$ ). Thus $a_{-} \neq 1$ and $\mathcal{K} \subseteq \mathcal{J}$. Moreover, $\bigvee \mathcal{K}=1$ because $h(\bigvee \mathcal{X})=1$, so if $b \in \mathcal{J}$ then $b \leqslant k$, for some $k \in \mathcal{K}$ (else $b_{-} \geqslant \bigvee \mathcal{K}=1$ ). But $b$ is nonzero and the elements of $\mathcal{K}$ are atoms, so this implies $b=k$. Thus $\partial \subseteq \mathcal{K}$, and they are equal.

As $\bigwedge_{a \in \mathfrak{Z}} h\left(a_{-}\right)$is the meet of the coatoms of $\mathcal{B}$, it is zero, and hence $\bigwedge \partial_{-}=0$ in $\mathcal{L}$. By Lemma $20, \mathcal{L}$ is a $\mathcal{J}$-lattice.

COROLlaRY 23. Every d-lattice is complemented. 
Indeed, if $h: \mathcal{L} \rightarrow \mathcal{B}$ is a collapsing homomorphism onto a complete atomic Boolean algebra, and $u \in \mathcal{B}$, then every element of $h^{-1}(u)$ is a complement of every element of $h^{-1}\left(u^{\prime}\right)$, where $u^{\prime}$ denotes the Boolean complement of $u$.

Recall that an element $c$ of a complete lattice is compact if $c \leqslant \bigvee X$ implies $c \leqslant \bigvee F$, for some finite subset $F \subseteq X$. A complete lattice $\mathcal{L}$ is algebraic if every element of $\mathcal{L}$ is a join of compact elements. For example, every finite lattice is algebraic. The ideal lattice of any lattice is algebraic.

THEOREM 24. If a $\mathcal{J}$-lattice $\mathcal{L}$ satisfies any one of the following conditions, then it is a complete atomic Boolean algebra.

1. $\mathcal{L}$ is uniquely complemented.

2. $\mathcal{L}$ is atomic, that is, every element is a join of atoms.

3. $\mathcal{L}$ is modular.

4. $\mathcal{L}$ is semimodular and algebraic.

Proof: The first two are easy to prove (using Theorem 22).

For $(3)$, let $\mathcal{L}$ be a $\mathcal{J}$-lattice and suppose that the map $h: \mathcal{L} \rightarrow \mathcal{B}$ as in Theorem 22 is not an isomorphism. Then there exists an element $u \in \mathcal{B}$ such that $\left|h^{-1}(u)\right|>1$. Let $a$ be the greatest element of $h^{-1}(u)$ and let $b$ be the least element. Note that $b<a$. Let $c \in h^{-1}\left(u^{\prime}\right)$. Then $a$ and $b$ are complements of $c$, and hence $\{0, a, b, c, 1\}$ forms a pentagon. Thus $\mathcal{L}$ is nonmodular.

For (4), let $\mathcal{L}$ be a $\mathcal{J}$-lattice which is semimodular and algebraic. By a simple modification of the proof of Theorem 18, we can show that every compact element of $\mathcal{L}$ is a join of atoms, so that $\mathcal{L}$ is atomic, and hence Boolean by (2). The crucial observation is that because the join of the atoms of $\mathcal{L}$ is 1 , every compact element is less than or equal to the join of finitely many atoms.

We finish by constructing an example of a semimodular $\partial$-lattice which is not Boolean. By Theorem 24(4), such a lattice must be infinite.

Let $X, Y$ be disjoint infinite sets not containing $z$, and let $W=X \dot{U} Y \dot{\cup}\{z\}$. Define $\mathcal{S}$ to be the collection of all subsets $A \subseteq W$ which satisfy the following conditions.

(i) If $A \cap Y$ is infinite, then $z \in A$.

(ii) If $A \cap Y$ is finite and $X \backslash A$ is infinite, then $z \notin A$.

Note that if $A \cap Y$ and $X \backslash A$ are both finite, then $z$ is allowed to be in $A$ or not. Order $\mathcal{S}$ by set inclusion.

THEOREM 25. The ordered set $\mathcal{S}$ is a semimodular $\partial$-lattice, but is not an atomic Boolean algebra.

Proof: $\mathcal{S}$ is not a sublattice of $\mathfrak{P}(W)$, so we need to proceed carefully.

Claim 1. $\{A \in \mathcal{S}: z \in A\}$ is an order filter of $\mathfrak{P}(W)$. For if $z \in A$, then by the contrapositive of (ii) either $A \cap Y$ is infinite or $X \backslash A$ is finite. Thus $B \supseteq A \in \mathcal{S}$ implies 
that $B \cap Y$ is infinite or $X \backslash B$ is finite, and of course $z \in B$. Thus $B$ satisfies (ii), and (i) vacuously, so $B \in \mathcal{S}$.

ClaIm 2. $\{A \in \mathcal{S}: z \notin A\}$ is an ideal of $\mathfrak{P}(W)$. For if $z \notin A$, then by the contrapositive of (i), $A \cap Y$ is finite. Thus $B \subseteq A \in \mathcal{S}$ implies $B \in \mathcal{S}$, similarly to Claim 1. Likewise, if $z \notin A_{1}, A_{2}$, both in $\mathrm{S}$, then $A_{1} \cap Y$ and $A_{2} \cap Y$ are both finite. Thus $\left(A_{1} \cup A_{2}\right) \cap Y$ is finite, and of course $z \notin A_{1} \cup A_{2}$, whence $A_{1} \cup A_{2} \in \mathcal{S}$.

Claim 3. $S$ is a complete lattice. Indeed, it is not hard to see that the operations are given as follows.

$$
\begin{aligned}
& \bigvee A_{i}= \begin{cases}\bigcup A_{i} \cup\{z\}, & \text { if } \bigcup A_{i} \cap Y \text { is infinite, } \\
\bigcup A_{i}, & \text { otherwise. }\end{cases} \\
& \bigwedge A_{i}= \begin{cases}\bigcap A_{i} \backslash\{z\}, & \text { if } \bigcap A_{i} \cap Y \text { is finite and } X \backslash \cap A_{i} \text { is infinite, } \\
\bigcap A_{i}, & \text { otherwise. }\end{cases}
\end{aligned}
$$

Note that by Claims 1 and 2, for any pair of sets $A \vee B=A \cup B$, and $A \wedge B=A \cap B$ except when $z \in A \cap B \notin S$.

Claim 4. $\mathcal{S}$ is a J-lattice. The atoms of $\mathcal{S}$ are the singletons $\{x\}$ with $x \in X \cup Y$. The join of all the atoms is $W$, and the join of all the atoms except $\{x\}$ is $\{x\}_{-}=W \backslash\{x\}$. From these observations it follows that $\partial=\{\{x\}: x \in X \cup Y\}$. Now check properties (1)-(4) for a $\partial$-lattice (using the definitions of meet and join above).

Claim 5. $S$ is semimodular. Let $A$ and $C$ be incomparable, and let $A>B>A \wedge C$. If perchance $z \in B$, put $D=C$. Then $A \wedge(B \vee C)=A \cap(B \cup C)=B$, as desired. So we may assume that $z \notin B$, and hence $z \notin A \wedge C$.

If $C=(A \cap C) \cup\{z\}$, then $z \notin A$. In this case again take $D=C$, and $A \wedge(B \vee C)=$ $A \cap(B \cup C)=B$.

Otherwise, there is an element $x \in X \cup Y$ in $C-(A \cap C)$. Let $D=(A \cap C) \cup\{x\}$. Then $z \notin B \cup D$, so the operations are again setwise and $A \wedge(B \vee D)=B$.

Claim 6. $\mathcal{S}$ is not an atomic Boolean algebra. In fact, the sets $X, X \cup\{z\}, Y, W$ and $\emptyset$ form a pentagon.

\section{REFERENCES}

[1] H.-J. Bandelt, 'On complete distributivity and maximal $d$-intervals in complete lattices', in Colloq. Math. Soc. János Bolyai 14, Lattice Theory (North-Holland Publishing Co., Amsterdam, 1976), pp. 29-43.

[2] H.-J. Bandelt, 'Tight residuated mappings and d-extensions', in Colloq. Math. Soc. János Bolyai 29, Universal Algebra (North-Holland Publishing Co., Amsterdam, 1982), pp. 61-72. 
[3] R. Freese and J.B. Nation, 'Covers in free lattices', Trans. Amer. Math. Soc. 288 (1985), 1-42.

[4] P.R. Halmos, 'Reflexive lattices of subspaces', J. London Math. Soc. 4 (1971), 257-263.

[5] A. Hopenwasser, 'Complete distributivity', in Operator theory: Operator algebras and applications, Proc. Symp. Pure Math. 51 (Amer. Math. Soc., Providence, RI, 1990), pp. 285-305.

[6] A. Katavolos and E. Katsoulis, 'Semisimplicity in operator algebras and subspace lattices', J. London Math. Soc. 42 (1990), 365-372.

[7] W.E. Longstaff, 'Strongly reflexive lattices', J. London Math. Soc. 1 (1975), 491-498.

[8] W.E. Longstaff, 'Remarks on semisimple reflexive algebras', in Proc. Conf. Automatic Continuity and Banach Algebras, (R.J. Loy, Editor), Centre for Math. Anal. 21 (A.N.U., Canberra, Australia, 1989), pp. 273-287.

[9] W.E. Longstaff, 'A note on the semisimplicity of reflexive operator algebras', in Proc. Int. Workshop on Analysis and its Applic., 4th Annual Meeting (Dubrovnik-Kupari, Yugoslavia, 1990), pp. 45-50.

[10] W.E. Longstaff and P. Rosenthal, 'On two questions of Halmos concerning subspace lattices', Proc. Amer. Math. Soc. 75 (1979), 85-86.

[11] O. Panaia, Quasi-spatiality of isomorphisms for certain classes of operator algebras, (Ph.D. Thesis) (University of Western Australia, 1995).

[12] G.N. Raney, 'Completely distributive complete lattices', Proc. Amer. Math. Soc. 3 (1952), 677-680.

[13] G.N. Raney, 'Tight Galois connections and complete distributivity', Trans. Amer. Math. Soc. 97 (1960), 418-426.

Department of Mathematics

The University of Western Australia

Nedlands, WA 6907

Australia

e-mail: longstaf@maths.uwa.edu.au oreste@maths.uwa.edu.au
Department of Mathematics

University of Hawaii

Honolulu, HI 96822-2273

United States of America

e-mail: jb@math.hawaii.edu 